\section{Ovarial-Ca.: schlechtere Prognose bei Gewichtsverlust während der Chemotherapie}

\author{
Übergewicht gilt auch bei der Entwicklung eines epithelialen Ovarial- \\ karzinoms (EOC) als Risikofaktor, aber die Gewichtsabnahme während \\ der Chemotherapie ist dann meist ein schlechtes Zeichen.
}

\begin{abstract}
n einer retrospektiven Analyse von 190
EOC-Krankheitsverläufen zwischen 2008 und 2013 wurde der Zusammenhang zwischen Gewichtsabnahme während der Erstlinienchemotherapie und Prognose überprüft. Die meisten Frauen hatten ein fortgeschrittenes High-GradeEOC und waren komplett oder bestmöglich reseziert worden.

Während sich in frühen Stadien keine signifikanten Unterschiede im Körpergewicht im Verlauf der Chemotherapie zeigten, ergaben sich bei Frauen in fortgeschrittenen Stadien signifikante Schwankungen, die mit dem progressionsfreien Überleben (PFS) korrelierten. EOC-Patientinnen, die innerhalb der
\end{abstract}

ersten sechs Monate mehr als $5 \%$ ihres Gewichts verloren oder mehr als $5 \%$ gegenüber ihrem ursprünglichen Körpergewicht zugelegt hatten, zeigten ein schlechteres bzw. längeres Überleben als Patientinnen mit weitgehend konstantem Gewicht (6 vs. 13 vs. 15 Monate).

Patientinnen im fortgeschrittenen Stadium zeigten darüber hinaus signifikante Differenzen beim Gesamtüberleben (OS) in Abhängigkeit von der Gewichtsveränderung - mit 24,3, 42,4 und 66,2 Monaten. In der multivariaten CoxAnalyse ergab sich eine signifikante Beeinflussung des PFS bzw. des OS durch Gewichtsveränderungen vom ersten bis zum sechsten CT-Zyklus (PFS: Hazard
Ratio [HR] 0,97; 95\%-Konfidenzintervall [95\%-KI] 0,95-0,99; OS: HR 0,94, $95 \%$-KI 0,91-0,97). Das OS korrelierte darüber hinaus auch mit Gewichtsveränderungen vom dritten bis zum sechsten Zyklus (HR 0,93, 95 \%-KI 0,88-0,98).

Fazit: Frauen mit einem fortgeschrittenen Ovarialkarzinom, die während der Erstlinienchemotherapie mehr als $5 \%$ Gewicht verloren, hatten ein verschlechtertes PFS und OS. Eine Gewichtszunahme um mehr als $5 \%$ verbesserte die Prognose.

Bei Patientinnen in frühen Stadien wurde dagegen kein derartiger Zusammenhang gesehen. Inwieweit diese $\mathrm{Zu}-$ sammenhänge von weiteren Parametern wie körperliche Aktivität oder Ernährung beeinflusst werden, konnte in dieser Beobachtungsstudie nicht ermittelt werden.

Barbara Kreutzkamp

Mardas M et al. Body weight changes in patients undergoing chemotherapy for ovarian cancer influence progression-free and overall survival. Support Care Cancer. 2017;25(3):795-800

\title{
US-Epidemiologen warnen vor steigendem Rohmilch-Konsum
}

\section{Krankheitsausbrüche in Zusammenhang mit dem Konsum von Kuh- milch(produkten) sind nach US-Daten fast immer durch Rohmilch bedingt.}

$\mathrm{D}$ er Wunsch nach einer „natürlichen“ Ernährungsweise mit Lebensmitteln, die möglichst nicht industriell verarbeitet sind, führt auch in den USA dazu, dass Milch, so wie sie aus dem Kuheuter fließt, immer beliebter wird.

Sollte sich dieser Trend fortsetzen, ist mit einem beträchtlichen Anstieg von Krankheiten durch kontaminierte Kuhmilch(produkte) zu rechnen. Das zeigt eine Analyse in „Emerging Infectious Diseases", einem Publikationsorgan der Centers of Disease Control. Die Analyse basiert auf den Daten aller Krankheitsausbrüche in den USA von 2009-2014, die durch Verunreinigung von Kuhmilch oder -käse mit EHEC, Salmonella spp., Listeria monocytogenes oder Campylobacter spp. ausgelöst worden waren. Im Durchschnitt gab es im Rahmen solcher Ausbrüche pro Jahr 761 Erkrankungen und 22 Krankenhauseinweisungen. Obwohl Rohmilch bzw. Rohmilchkäse nur bei etwa 3,2\% bzw. 1,6\% der Bevölkerung auf dem Speiseplan stehen, waren sie ursächlich für $96 \%$ der Erkrankungen. Krankheitsfälle durch EHEC, Salmonella spp. und Campylobacter spp. gingen häufiger auf unbehandelte als auf pasteurisierte Milch zurück. Listerieninfektionen wurden dagegen überraschenderweise häufiger bei den Erkrankungen gefunden, die durch erhitzte Milch verursacht waren. Das Risiko für eine
Erkrankung durch eines der vier Pathogene ist demnach bei Rohmilchkonsumenten 840-mal so hoch wie bei Konsumenten von erhitzter Milch, das für Krankenhausaufnahmen um den Faktor 45 erhöht.

Die Studienautoren warnen: Sollte der Rohmilchkonsum weiter steigen, sei eine erhebliche Zunahme der genannten Erkrankungen zu erwarten. So würde die Zahl der Krankheitsfälle bei einer Verdopplung der Konsumentenzahl ebenfalls um $100 \%$ zunehmen, wobei Infektionen mit Salmonellen und Campylobacter spp. dominierten. Die Autoren betonen auch, dass ihre Daten die reale Situation wahrscheinlich unterschätzen, da sie sich auf die Auswertung von Ausbrüchen beschränken mussten. Die Zahl sporadischer Erkrankungen durch kontaminierte Rohmilchprodukte sei wahrscheinlich wesentlich höher.

\section{Dr. Beate Schumacher}

Costard S et al. Outbreak-Related Disease Burden Associated with Consumption of Unpasteurized Cow's Milk and Cheese, United States, 2009-2014. Emerging Infectious Diseases 2017; Volume 23, Number 6 